\title{
ON THE COEFFICIENTS OF MEROMORPHIC UNIVALENT FUNCTIONS
}

\author{
D. K. THOMAS
}

ABSTRACT. Let $f \in \Sigma$; the class of all analytic univalent functions defined in $\gamma=\{z:|z|>1\}$. For $f, g \in \Sigma$ define $h$ in $\gamma$ by $h(z)=f(z)^{1-\alpha} g(z)^{\alpha}$, $0<a<1$. If $h(z)=z+\sum_{n=0}^{\infty} c_{n} z^{-n}$, it is shown that $\sum_{n=1}^{\infty} n\left|c_{n}\right|^{2}<\infty$. This result is used to show that if $B_{\alpha}$ denotes the class of all me romorphic Bazilevič functions of type $a$ and $f \in B_{\alpha}$ with $f(z)=z+\sum_{n=0}^{\infty} a_{n} z^{-n}$, then $n a_{n}=O(1)$ as $n \rightarrow \infty$, the result being best possible.

Denote by $\Sigma$ the class of all analytic univalent functions $f$ defined in $\gamma=\{z:|z|>1\}$ by the power series $f(z)=z+\sum_{n=0}^{\infty} a_{n} z^{-n}$. Then, as is well known,

$$
\sum_{n=1}^{\infty} n\left|a_{n}\right|^{2} \leq 1,
$$

which implies that $a_{n}=o(1) n^{-1 / 2}$ as $n \rightarrow \infty$. Clunie and Pommerenke [3] have shown that this result is not best possible and that $a_{n}=O(1) n^{-1 / 2-1 / 300}$ as $n \rightarrow \infty$. In the other direction, Clunie [1] has given an example of a function $f \in \sum$ satisfying $n a_{n}>n^{\delta}$ for $\delta>0$, and infinitely many $n$. The correct index of $n$, the so-called Clunie constant, is unknown.

For various subclasses of $\Sigma$ the situation is much better. For example, if $\Sigma^{*}$ denotes the class of all meromorphic starlike functions defined in $\gamma$, then Clunie [2] has shown that $a_{n}=O(1) n^{-1}$ as $n \rightarrow \infty$, and that the index of $n$ is best possible. The same result has been proved by Pommerenke [6] for meromorphic close-to-convex functions. In fact Pommerenke was able to show that $a_{n}=O(1) n^{-1}$ holds for functions $f \in \Sigma$ satisfying $\operatorname{Re}\left(z f^{\prime}(z) / g(z)\right)>0$ for $z \in \gamma$ with $g \in \Sigma, g(z) \neq 0$.

In [7], the following subclasses of $\Sigma$ were introduced. Let $f \in \Sigma$, $g \in \Sigma^{*}$ and for real $\alpha$ let $\operatorname{Re}\left(z f^{\prime}(z) / f(z)^{1-\alpha} g(z)^{\alpha}\right)>0$ for $z \in \gamma$. Then $f$ defined in this way is called a meromorphic Bazilevič function of type $\alpha$. We denote these classes by $B(\alpha)$, and note that $B(0)$ and $B(1)$ are the classes of meromorphic starlike and meromorphic close-to-convex functions

Received by the editors August 27, 1973.

AMS (MOS) subject classifications (1970). Primary 30A32, 30A34, 30A36. 
respectively. In [7], the coefficient problem for $B(\alpha)$ was considered, and it was shown that if $f \in B(\alpha)$, with $f(z)=z+\sum_{n=0}^{\infty} a_{n} z^{-n}, 0 \leq \alpha \leq 1$, and $f$ and $g$ have no zeros in $\gamma$, then $n a_{n}=O(1)(\log n)^{1 / 2}$ as $n \rightarrow \infty$. We shall show that the logarithmic factor can be removed, giving the best possible estimate $n a_{n}=O(1)$ as $n \rightarrow \infty$. We prove in fact:

Theorem 1. Let $f, g \in \Sigma$, with $f(z)=z+\Sigma_{n=0}^{\infty} a_{n} z^{-n}$, and suppose that both $f$ and $g$ have no zeros in $\gamma$. Then if for $0 \leq \alpha \leq 1$

$$
\operatorname{Re} \frac{z f^{\prime}(z)}{f(z)^{1-\alpha} g(z)^{a}}>0
$$

for $z \in \gamma, n a_{n}=O(1)$ as $n \rightarrow \infty$.

Note that the result is proved for a wider class than $B(\alpha)$, since $g$ need not necessarily belong to $\Sigma^{*}$. We do however still require that $f$ and $g$ have no zeros in $\gamma$.

In order to prove Theorem 1 , we require the following result, which is of interest in itself.

Theorem 2. Let $f, g \in \Sigma$ with

$$
f(z)=z+\sum_{n=0}^{\infty} a_{n} z^{-n} \quad \text { and } \quad g(z)=z+\sum_{n=0}^{\infty} b_{n} z^{-n} .
$$

For $0<a<1$, and forsufficientlylarge $z$, define the function $h$ in $y$ by

$$
h(z)=f(z)^{1-a_{g}(z)^{\alpha}}=z+\sum_{n=0}^{\infty} c_{n} z^{-n} .
$$

Then $\sum_{n=1}^{\infty} n\left|c_{n}\right|^{2}<\infty$.

We need the following area principle due to Grunsky [4].

Lemma. Let the function $k$ be defined for $z \in \gamma$ by $k(z)=\sum_{n=0}^{m} d_{n} z^{-n}+$ $\sum_{n=-\infty}^{-1} d_{n} z^{n}$, and suppose that $d_{m} \neq 0$. If $k$ is strongly m-valent in $\gamma$, that $i s, k$ assumes in $\gamma$ no value more than $m$ times, then

$$
\sum_{n=-\infty}^{-1}|n|\left|d_{n}\right|^{2} \leq \sum_{n=1}^{m} n\left|d_{n}\right|^{2} \text {. }
$$

Proof of Theorem 2. A simple limiting argument shows that in order to prove the result, it is sufficient, provided the bound obtained for $\sum_{n=1}^{\infty} n\left|c_{n}\right|^{2}$ is independent of $\alpha$, to consider $\alpha$ to be rational.

Assume then, that $\alpha=p / q$ where $p, q$ are positive integers with $p<q$. Since $f$ and $g$ have no zeros in $\gamma$, we can define $F$ and $G$ in $\Sigma$ by 
$F(z)=f\left(z^{q}\right)^{1 / q}$ and $G(z)=g\left(z^{q}\right)^{1 / q}$. Further let $\mathcal{F}$ and $\mathcal{S}$ be defined for $z \in \gamma$ by $\mathcal{F}(z)=F(z)^{q-p}$ and $\mathcal{G}(z)=G(z)^{p}$ respectively. Then for $z \in \gamma$ we can write

$$
\mathcal{F}(z)=z^{q-p}+\sum_{n=1}^{\infty} A_{n} z^{-n q+q-p}, \quad \varrho(z)=z^{p}+\sum_{n=1}^{\infty} B_{n} z^{-n q+p} .
$$

Clearly $\mathcal{F}$ is strongly $q-p$-valent and $@$ strongly $p$-valent in $\gamma$. Thus by the Lemma,

$$
\sum_{n=1}^{\infty}(n q+p-q)\left|A_{n}\right|^{2} \leq q-p
$$

and

$$
\sum_{n=1}^{\infty}(n q-p)\left|B_{n}\right|^{2} \leq p
$$

Next define the function $H$ in $\gamma$ by $H(z)=\mathscr{F}(z) \mathscr{G}(z)$. Then for $z \in \gamma$ we can write $H(z)=z^{q}+\Sigma_{n=0}^{\infty} C_{n} z^{-n q}$. Now $H^{\prime}(z)=\mathcal{F}^{\prime}(z) \mathscr{S}(z)+\mathcal{F}(z) \mathfrak{S}^{\prime}(z)$, and so by the Schwarz inequality

$$
\left|H^{\prime}(z)\right|^{2} \leq\left(|\mathfrak{F}(z)|^{2}+\mid \mathscr{G}_{(z)}\left({ }^{2}\right)\left(\left|\mathfrak{F}^{\prime}(z)\right|^{2}+\left|\mathcal{G}^{\prime}(z)\right|^{2}\right) .\right.
$$

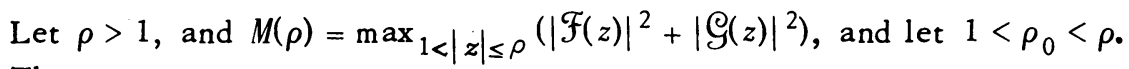
Then

$$
\int_{\rho_{0}}^{\rho} \int_{0}^{2 \pi}\left|H^{\prime}\left(r e^{i \theta}\right)\right|^{2} r d r d \theta \leq M(\rho) \int_{\rho_{0}}^{\rho} \int_{0}^{2 \pi}\left(\left|\mathcal{F}^{\prime}(z)\right|^{2}+\left|\mathcal{G}^{\prime}(z)\right|^{2}\right) r d r d \theta,
$$

and so

$$
\begin{aligned}
& q \rho^{2 q}-\sum_{n=1}^{\infty} n q\left|C_{n}\right|^{2} \rho^{-2 n q}-\left\{q \rho_{0}^{-2 q}-\sum_{n=1}^{\infty} n q\left|C_{n}\right|^{2} \rho_{0}^{-2 n q}\right\} \\
& \leq M(\rho) {\left[(q-p) \rho^{2(q-p)}-\sum_{n=1}^{\infty}(n q-q+p)\left|A_{n}\right|^{2} \rho^{-2(n q-q+p)}\right.} \\
&-\left\{(q-p) \rho_{0}^{2(q-p)}-\sum_{n=1}^{\infty}(n q-q+p)\left|A_{n}\right|^{2} \rho_{0}^{-2(n q-q+p)}\right\} \\
&+p \rho^{2 p}-\sum_{n=1}^{\infty}(n q-p)\left|B_{n}\right|^{2} \rho^{-2(n q-p)}\left.-\left\{p \rho_{0}^{2 p}-\sum_{n=1}^{\infty}(n q-p)\left|B_{n}\right|^{2} \rho_{0}^{-2(n q-p)}\right\}\right] .
\end{aligned}
$$


Using (3) and (4) we see that letting $\rho_{0} \rightarrow 1$

$$
\begin{aligned}
& q \rho^{2 q}-\sum_{n=1}^{\infty} n q\left|C_{n}\right|^{2} \rho^{-2 n q}-\left\{q-\sum_{n=1}^{\infty} n q\left|C_{n}\right|^{2}\right\} \\
& \leq M(\rho)\left[(q-p) \rho^{2(q-p)}+p \rho^{2 p}\right] .
\end{aligned}
$$

Let $|f(z)| \leq K$ and $|g(z)| \leq K$ for $1<|z| \leq 2$, and choose $\rho=2^{1 / q}$. Then $M(\rho)=M\left(2^{1 / q}\right) \leq K^{2(q-p) / q}+K^{2 p / q} \leq 2 K^{2}$, since $K \geq 1$. Thus (5) gives

$$
\begin{aligned}
& 4 q-\sum_{n=1}^{\infty} n q\left|C_{n}\right|^{2} 4^{-n}-\left(q-\sum_{n=1}^{\infty} n q\left|C_{n}\right|^{2}\right) \\
& \leq 2 K^{2}\left[(q-p) 4^{(q-p) / q}+p 4^{p / q}\right] \leq 16 K^{2} q,
\end{aligned}
$$

and so

$$
\sum_{n=1}^{\infty} n\left|C_{n}\right|^{2}\left(1-4^{-n}\right) \leq 16 K^{2}-3
$$

which gives $\Sigma_{n=1}^{\infty} n\left|C_{n}\right|^{2} \leq(4 / 3)\left(16 K^{2}-3\right)$. Finally note that $H\left(z^{1 / q}\right)=$ $F\left(z^{1 / q}\right)^{q-p}, G\left(z^{1 / q}\right)^{p}=f(z)^{1-a} g(z)^{a}$, and so $C_{n}=c_{n}$ for $n \geq 1$, and the result follows.

It remains only to establish the result when $f$ and $g$ have a common zero in $\gamma$. Suppose then that $f(\zeta)=g(\zeta)=0$ with $\zeta \in \gamma$. Then for $1<|z|<$ $(1+|\zeta|) / 2,1 / K<|f(z)|,|g(z)| \leq K$ for some $K>0$. Now

$$
h^{\prime}(z)=(1-\alpha) f^{\prime}(z) f(z)^{-\alpha} g(z)^{\alpha}+\alpha g^{\prime}(z) f(z)^{1-\alpha} g(z)^{\alpha-1},
$$

and so for $1<|z| \leq(1+|\zeta|) / 2,\left|h^{\prime}(z)\right|^{2} \leq K_{1}\left(\left|f^{\prime}(z)\right|^{2}+\left|g^{\prime}(z)\right|^{2}\right)$, where $K_{1}$ is constant. Now let $\rho_{0}>1$; then as before,

$$
\begin{aligned}
\rho^{2}-\sum_{n=1}^{\infty} n\left|c_{n}\right|^{2} \rho^{-2 n}-\left\{\rho_{0}^{2}-\sum_{n=1}^{\infty} n\left|c_{n}\right|^{2} \rho_{0}^{-2 n}\right\} \\
\leq K_{1}\left[\rho^{2}-\sum_{n=1}^{\infty} n\left|a_{n}\right|^{2} \rho^{-2 n}-\left(\rho_{0}^{2}-\sum_{n=1}^{\infty} n\left|a_{n}\right|^{2} \rho_{0}^{2 n}\right)\right. \\
\left.\quad+\rho^{2}-\sum_{n=1}^{\infty} n\left|b_{n}\right|^{2} \rho^{-2 n}-\left(\rho_{0}^{2}-\sum_{n=1}^{\infty} n\left|b_{n}\right|^{2} \rho_{0}^{-2 n}\right)\right] .
\end{aligned}
$$

Hence, letting $\rho_{0} \rightarrow 1$, and using (1), we have

$$
\sum_{n=1}^{\infty} n\left|c_{n}\right|^{2}\left(1-\rho^{-2 n}\right) \leq 1+\rho^{2}\left(2 K_{1}-1\right),
$$


and so

$$
\sum_{n=1}^{\infty} n\left|c_{n}\right|^{2} \leq \frac{\rho^{2}}{\rho^{2}-1}\left[1+\rho^{2}\left(2 K_{1}-1\right)\right] \leq K_{2}
$$

on choosing $\rho=(1+|\zeta|) / 2$. This proves Theorem 2 .

Proof of Theorem 1. Since $f$ and $g$ have no zeros in $\gamma$, the function $h$, defined in $\gamma$ by $h(z)=f(z)^{1-\alpha} g(z)^{\alpha}$ is analytic in $\gamma$. Thus from (2) we may write

$$
z f^{\prime}(z)=h(z) p(z),
$$

where $p$ is analytic in $\gamma, p(\infty)=1$ and $\operatorname{Re} p(z)>0$ for $z \in \gamma$. From (6) we thus have

$$
z-\sum_{k=1}^{\infty} k a_{k} z^{-k}=\left(z+\sum_{k=0}^{\infty} c_{k} z^{-k}\right)\left(1+\sum_{k=1}^{\infty} p_{k} z^{-k}\right),
$$

and so for $n \geq 1$,

$$
-n a_{n}=p_{n+1}+\sum_{\nu=0}^{n-1} c_{\nu} p_{n-\nu}+c_{n} .
$$

Cauchy's theorem gives for $k \geq 1$,

$$
p_{k}=\frac{r^{k}}{\pi} \int_{0}^{2 \pi} \operatorname{Re} p\left(r e^{i \theta}\right) e^{i k \theta} d \theta
$$

so that in (7) we have for $n \geq 1$,

$$
\begin{aligned}
-n a_{n} & =\frac{1}{\pi} \int_{0}^{2 \pi} \operatorname{Re} p\left(r e^{i \theta}\right)\left(r^{n+1} e^{i(n+1) \theta}+\sum_{\nu=0}^{n-1} c_{\nu} r^{n-\nu} e^{i(n-\nu) \theta}\right) d \theta+c_{n} \\
& =\frac{r^{n}}{\pi} \int_{0}^{2 \pi} \operatorname{Re} p\left(r e^{i \theta}\right)\left(h\left(r e^{i \theta}\right)-\sum_{\nu=n}^{\infty} c_{\nu}\left(r e^{i \theta}\right)^{-\nu} e^{i n \theta}\right) d \theta+c_{n} .
\end{aligned}
$$

Thus, since $\int_{0}^{2 \pi} \operatorname{Re} p\left(r e^{i \theta}\right) d \theta=2 \pi$,

$$
\begin{aligned}
n\left|a_{n}\right| & \leq \frac{r^{n}}{\pi}\left|\int_{0}^{2 \pi} \operatorname{Re}\left(r e^{i \theta}\right) h\left(r e^{i \theta}\right) e^{i n \theta} d \theta\right|+2 r^{n} \sum_{\nu=n}^{\infty}\left|c_{\nu}\right| r^{-\nu}+\left|c_{n}\right| \\
& \leq \frac{r^{n}}{\pi}\left|\int_{0}^{2 \pi} \operatorname{Re} p\left(r e^{i \theta}\right) b\left(r e^{i \theta}\right) e^{i n \theta} d \theta\right|+2 r^{n}\left(\sum_{\nu=n}^{\infty} \nu\left|c_{\nu}\right|^{2}\right)^{1 / 2}\left(\sum_{\nu=n}^{\infty} \frac{1}{\nu r^{2 \nu}}\right)^{1 / 2}+\left|c_{n}\right|
\end{aligned}
$$

by the Schwarz inequality. Now since $f$ and $g$ have no zeros in $\gamma,|f(z)|$, $|g(z)| \leq r+3[5]$, and so $|h(z)| \leq r+3$. Also since by Theorem 2, $\sum_{\nu=1}^{\infty} \nu\left|c_{\nu}\right|^{2}<\infty,\left|c_{n}\right| \leq K$, and if $\epsilon_{n}^{2}=\Sigma_{\nu=n}^{\infty} \nu\left|c_{\nu}\right|^{2}$, then $\epsilon_{n}^{2} \leq K$ and $\epsilon_{n} \rightarrow 0$ 
as $n \rightarrow \infty$. Thus

$$
n\left|a_{n}\right| \leq 2(r+3) r^{n}+2 \epsilon_{n}\left(n\left(1-1 / r^{2}\right)\right)^{-1 / 2}+K,
$$

and so for $n \geq 2$, choosing $r=(1-1 / n)^{-1 / 2}$, we have $n a_{n}=O(1)$ as $n \rightarrow \infty$.

\section{REFERENCES}

1. J. G. Clunie, On schlicht functions, Ann. of Math. (2) 69 (1959), 511-519. MR $21 \# 6438$.

2. , On meromorphic schlicht functions, J. London Math. Soc. 34 (1959), 215-216. MR $21 \# 5737$.

3. J. G. Clunie and C. Pommerenke, On the coefficients of univalent functions, Michigan Màth. J. 14 (1967), 71-78. MR 34 \#7786.

4. H. Grunsky, Koeffizientenbedingungen für schlicht abbildende meromorphe Funktionen, Math. Z. 45 (1939), 29-61.

5. G. Pólya and G. Szegö, Aufgaben und Lehrsätze aus der Analysis, Springer, Berlin, 1925, p. 25; photographic reproduction, Vol. II, Dover, New York, 1945. MR 7, 418.

6. C. Pommerenke, Über einige Klassen meromorpher schlichter Funktionen, Math. Z. 78 (1962), 263-284. MR $28 \# 1285$.

7. D. K. Thomas, On Bazilevi ̌̌ functions, Trans. Amer. Math. Soc. 132 (1968), 353-361. MR $36 \# 5330$.

DEPARTMENT OF PURE MATHEMATICS, UNIVERSITY COLLEGE, SWANSEA, WALES 